\title{
Kinesthetically guided reaching accuracy in individuals with a history of traumatic anterior shoulder dislocation
}

\author{
You-jou Hung' \\ Warren G Darling ${ }^{2}$ \\ 'Doctor of Physical Therapy \\ Program, Department of Nursing \\ and Rehabilitation Sciences, Angelo \\ State University, San Angelo, TX, USA; \\ ${ }^{2}$ Department of Health and Human \\ Physiology, The University of lowa, \\ lowa City, IA, USA
}

This article was published in the following Dove Press journal:

Orthopedic Research and Reviews

29 May 2013

Number of times this article has been viewed
Background: The purpose of the study was to investigate whether individuals with a history of traumatic anterior shoulder dislocation show larger reaching errors than those with healthy shoulders and to determine if they implement different reaching strategies to protect the injured shoulder.

Methods: Ten people with a history of traumatic anterior shoulder dislocation and 15 with healthy shoulders volunteered for this study. After viewing targets in space, participants pointed with the unconstrained arm to remembered target locations in space without visual guidance. Nine different targets were located in various planes and heights. Endpoint reaching errors were determined by comparing the finger endpoint position without visual guidance to the target location. Shoulder rotation angle at the endpoint was also compared between groups.

Results: Participants with injured shoulders were able to point voluntarily to visually specified targets as accurately as participants with healthy shoulders ( $1 \mathrm{~cm}$ difference). However, participants with injured shoulders showed less shoulder external rotation (average $12^{\circ}$ difference) at the target location when compared with healthy shoulders. This difference was consistent over a large range of target locations.

Conclusion: Individuals with a history of traumatic anterior shoulder dislocation have sufficient kinesthetic information about their upper limb orientation to point accurately to visually specified targets in space. However, individuals with injured shoulders acquired a new motor strategy to reach with less shoulder external rotation, presumably to protect the injured shoulder from recurrent injuries.

Keywords: shoulder injuries, physiotherapy, shoulder instability, motor control

\section{Introduction}

The glenohumeral joint $(\mathrm{GHJ})$ is prone to instability because the glenoid fossa is a shallow structure that provides little bony constraint for the humeral head. After a dislocation, injured passive shoulder stabilizing structures (ie, the glenoid labrum, GHJ capsule, and glenohumeral ligaments) may not be able to provide sufficient mechanical restraint to the joint, especially in shoulder positions involving abduction with external rotation. ${ }^{1-4}$ It was suggested that altered neuromuscular control over shoulder girdle muscles due to compromised proprioceptive sensation may also contribute to recurrent shoulder instability. ${ }^{5-10}$ If awareness of shoulder joint orientation is impaired, the individual may not be able to engage appropriate muscle activation at positions in which voluntary contraction of shoulder muscles acting as active joint stabilizers is crucial for shoulder stability. At a functional level, the individual may not be able to move the upper extremity accurately to a desired target without visual 
feedback, or sense the exact location of the hand/fingers in space which is crucial for some activities (eg, releasing the ball during a pitch).

Statistically significant differences in shoulder joint position sense have been reported between individuals with healthy shoulders and those with a history of anterior shoulder dislocation using the "passive matching of passive positioning" protocols. ${ }^{6,9-12}$ However, the clinical and functional significance of such a deficit is questionable because the differences between injured and healthy shoulders were relatively small (0.6-2.3 degrees). ${ }^{6,9,10,12}$ Moreover, information from muscle spindles and other sources (eg, cutaneous receptors, Golgi tendon organs, sense of effort) during voluntary arm movement is also beneficial for joint position sense. ${ }^{13-20}$ Despite greater position sense errors being reported in unstable shoulders than in healthy shoulders with passive matching protocols, ${ }^{6,9-12}$ it is not clear if compromised shoulder stabilizers as well as passive position sense would have an impact on functional reaching accuracy.

Proper motor control of the hand in space is important for many daily upper limb activities (eg, reaching). Reaching accuracy to remembered visual targets in three dimensions has been studied in subjects without shoulder pathology $y^{21-25}$ and in subjects with neurological disorders. ${ }^{21,26}$ However, no previous study has examined kinesthetically guided reaching accuracy in individuals with a history of traumatic anterior shoulder dislocation using a functional testing protocol with shoulder, elbow, wrist, and finger motions unrestrained. In addition, it is also unknown whether individuals with a history of anterior shoulder dislocation would implement a different reaching strategy to protect their injured shoulders when reaching towards some vulnerable positions.

The purpose of the present study was to determine whether individuals with a history of traumatic anterior shoulder dislocation show larger reaching errors than individuals with healthy shoulders. The second aim of the study was to investigate whether individuals with injured shoulders used different reaching strategies from healthy controls while reaching to various target locations. Because information from muscle spindles and other sources during voluntary arm movement may provide accurate shoulder position sense, ${ }^{12}$ we hypothesized that individuals with injured and healthy shoulders would show similar kinesthetically guided reaching accuracy to visually specified targets in space. However, participants with injured shoulders might take advantage of redundancy to achieve similar reaching accuracy with different upper limb configurations.

\section{Materials and methods \\ Participants}

Ten people (seven men and three women, aged 19-37 years) with a history of traumatic anterior shoulder dislocation and 15 people ( 11 men and four women, aged 20-39 years) with healthy shoulders volunteered for this study. The human subject institutional review board of the University of Iowa approved the study, and all participants signed informed consent documents prior to participating in the study. Inclusion criteria for the participants with injured shoulders were: a minimum of one episode of complete traumatic anterior shoulder dislocation documented in their medical record, a positive apprehension test administered by the primary investigator, and no surgical repair prior to testing. In addition to the subject's medical record, the apprehension test (specificity $98.91 \%$, sensitivity $52.78 \%)^{27}$ was used to confirm anterior traumatic instability. People with multidirectional instability, degenerative arthritis, muscle weakness (weaker than "normal" with manual muscle testing), or inability to achieve the designated shoulder positions without pain or apprehension were excluded from the study. People with only partial dislocation or subluxation were also excluded from the study. The mechanism of injury was unexpected external force perturbation due to falls or sports injuries for the participants with a history of anterior GHJ dislocation, resulting in three subjects with one dislocation, four subjects with two dislocations, two subjects with three dislocations, and one subject with five prior dislocations. Six subjects injured their dominant arms (five right arms and one left arm) and four subjects injured their nondominant arms (four left arms). The time since their last dislocation ranged from four to 19 months. All participants with a history of GHJ dislocation were diagnosed and treated at the University of Iowa Hospitals and Clinics.

More individuals with healthy shoulders $(n=15)$ than with injured shoulders $(n=10)$ volunteered for the study. Based on pilot data from six individuals (three with injured shoulders and three with healthy shoulders), a sample size of 10 participants in the experimental group was estimated to be adequate to detect group differences with a power of $80 \%$ when the significance level was 0.05 . Inclusion criteria for the control group included no known history of shoulder injury requiring medical treatment and being able to move the shoulders to the target position without discomfort or limitation. Because gender ${ }^{28}$ and age ${ }^{8,29,30}$ may have an impact on joint position sense, participants with a similar gender ratio and age range were recruited for both the control and experimental groups. To reduce the potential effect of innate 
differences in proprioception between the two groups, the dominant arm of nine randomly chosen healthy subjects and the nondominant arm of the six remaining healthy subjects were examined to match the arm dominance ratio in subjects with injured shoulders (dominant to nondominant, 3:2) for all between-group comparisons. Participants with healthy shoulders were recruited from the University of Iowa student body and staff members.

\section{Measures}

The Ascension Technology miniBIRD (MB) electromagnetic tracking system (Ascension Technology Corp, Burlington, VT, USA) was used to record upper limb kinematics ( $74 \mathrm{~Hz}$ sampling rate). The validity of the system for motion analysis has been documented, ${ }^{31-33}$ and the positional and rotational errors measured were less than $2 \%$ of the range of motion when utilized within its optimal operating range between $22.5 \mathrm{~cm}$ and $64.0 \mathrm{~cm}$ from the transmitter. ${ }^{31}$ Five electromagnetic receivers of the MB system were secured on the skin over each participant's manubrium, the distal end of the scapular acromion, the lateral epicondyle of the humerus, the wrist cuff over the styloid process of the ulna, and the tip of the index finger. Participants sat in an armless chair with a vertical back support during all testing procedures.

\section{Procedure}

After giving their consent, all potential participants first attended a screening session (the first visit) prior to the data collection phase of the study. The primary investigator first examined whether participants could reach to all predetermined target locations freely without limitation, pain, or apprehension with both arms. Second, the primary investigator used manual muscle testing to examine whether participants had weakness of their shoulder external and internal rotators and flexors and abductors. At the beginning of testing, participants with their eyes closed were positioned to 0 degrees of shoulder abduction, 0 degrees of shoulder rotation, 90 degrees of shoulder flexion, and 90 degrees of elbow flexion measured with a goniometer. Subjects were also asked to keep their wrist in a neutral position with extended fingers. Shoulder angles were recorded with the MB system in this position (set as zero reference). The shoulder position of all following trials was compared with this standard position to determine shoulder angles.

Endpoint reaching accuracy was examined for nine different targets located in three different planes in which various shoulder abduction and rotation angles could be used to accomplish the task. These were the parasagittal plane through the GHJ of the moving limb, the frontal plane through the GHJ of the moving limb, and a vertically oriented oblique plane through the participant's GHJ that equally bisected the previous two planes (Figure 1). There were three targets in each of the three reference planes. The height of the three targets was determined by the height of each participant's anterior superior iliac spine (low target), GHJ (middle target), and the top of the head (top target) when measured from a seated position during the screening visit. Targets (in bright yellow) were $1.5 \mathrm{~cm} \times 1.5 \mathrm{~cm}$ in size with adhesive backing. Top, middle, and bottom targets were attached to a black tripod at various heights based on the body dimensions of the individual subject. The "horizontal" distance between the targets and the GHJ varied between the participants and was determined for each participant as two thirds of the distance from the index finger tip to the GHJ with the arm and fingers extended. Markers (squares with a circle center) were positioned on the ground to indicate the proper tripod location for each plane (Figure 1). To avoid use of visual feedback to remember the designated target location during

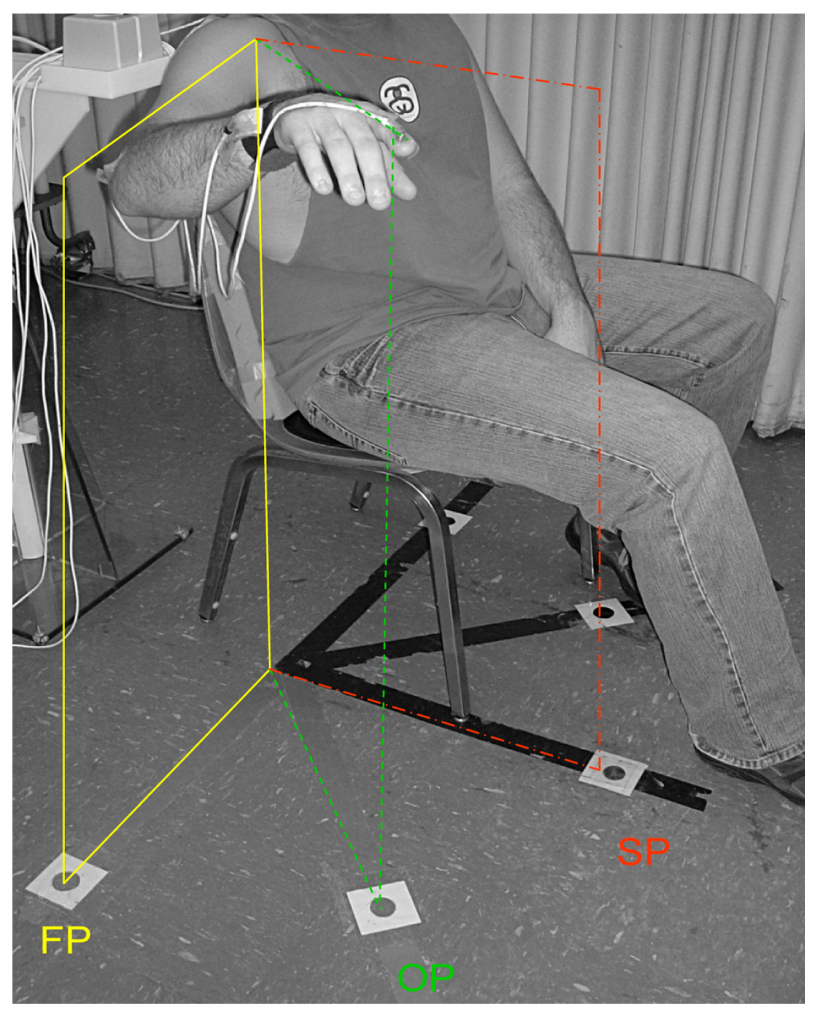

Figure I Kinesthetically guided reaching. Participants first located the target visually and then reached to the remembered location with their index fingertip without vision. Notes: The target for the trial shown here is the middle target (same height as the subject's GHJ) in the oblique plane. The three planes are the parasagittal plane through the $\mathrm{GH}$ of the moving limb (in red), the frontal plane through the $\mathrm{GHJ}$ of the moving limb (in yellow), and a vertically oriented oblique plane through the subject's GHJ that bisected the previous two planes equally (in green).

Abbreviation: $\mathrm{GHJ}$, glenohumeral joint. 
practice, participants were not allowed to practice during the investigator's demonstration and practiced later without receiving reaching feedback concerning pointing accuracy from the investigator. Moreover, the tripod with targets was removed immediately after viewing so that participants could not grope for the target and receive proprioceptive/cutaneous feedback about the target location.

At the starting position, participants were instructed to sit upright against a back support with the unsupported arm resting by their side. After the primary investigator verbally specified the target for the trial (top, middle, or bottom targets that were fixed on the tripod), the participants first located the target visually for a second and then immediately reached to the remembered three-dimensional location at a comfortable speed using the index fingertip with their eyes closed. Participants kept the index finger at the target location for a second before returning to the starting position. After the participant viewed the instructed target, the investigator moved the tripod away during the participant's reaching movement to prevent any tactile feedback indicating the target location. Participants performed the task one plane at a time with eight trials for each target in each plane. The orders of planes and targets were randomly assigned to each subject. After finishing the reaching tests in all three planes, participants reached to touch each target for one second with vision allowed. Fingertip position from these trials were used as the reference location to compute reaching errors when comparing with earlier reaching trials without vision.

\section{Data analysis}

Skill Technology's 6D Research software (Skill Technologies Inc, Phoenix, AZ, USA) was used to compute positions and orientations of the $\mathrm{MB}$ receivers, and a custom designed program converted the data into a format that was further analyzed with Datapac 2K2 software (Run Technologies, Mission Viejo, CA, USA). For kinesthetically guided reaching accuracy, endpoint fingertip position was compared with the reference position collected at the end of the experiment to determine reaching errors. The root mean square errors were computed to represent the overall reaching errors for group comparison. Shoulder rotation angles were also examined at the end of each reaching movement. They were computed by constructing a local humeral coordinate system followed by Cardan angle rotations about axes fixed to the humerus. Absolute position data from three noncollinear points (the acromion, elbow, and wrist receivers) were used to construct a local coordinate system for the humerus. The orientation of the humerus was then calculated by relating the local humerus coordinate system to the global system with a series of ordered rotations about the humeral axes: vertical axis (defines yaw angle), medial-lateral axis (defines elevation angle), and long axis of the humerus (defines internal/external rotation angle). For the current study, we only examined and reported shoulder external/internal rotation angle because shoulder external rotation is most likely to impose additional stress over the anterior shoulder capsule and potentially elicit changes of reaching strategy in subjects with anterior shoulder instability. Mean shoulder rotation angles of the eight trials to each target location were calculated and analyzed.

Three-way analysis of variance with one between-group factor (injured versus healthy shoulders) and two repeatedmeasures factors (three planes and three target positions) was used to analyze endpoint reaching accuracy and shoulder rotation angle (and its consistency with various target locations). Tukey's honestly significant difference test was used for post hoc testing of significant main and interaction effects. Because there were three levels (three targets) for the repeated-measure factors, adjustments in degrees of freedom using Greenhouse-Geisser correction were applied. The statistical significance level ( $P$ value) was set at 0.05 for all comparisons. All analyses were conducted using Statistica (StatSoft Inc, Tulsa, OK, USA).

\section{Results}

No statistically significant differences were found for kinesthetically guided reaching accuracy between the subjects with injured shoulders and those with healthy shoulders (Figure 2, $\left.F_{1,23}=1.03, P=0.321\right)$. Further analysis indicated that group $\times$ plane $\times$ height $\left(F_{4,92}=0.69, P=0.483\right)$, plane $\times$ height $\left(F_{4,92}=1.92, P=0.113\right)$, and group $\times$ height $\left(F_{2,46}=0.52, P=0.598\right)$ interactions were not significant. However, the group $\times$ plane interaction was statistically significant $\left(F_{2,46}=4.08, P=0.023\right)$. Subjects with a history of shoulder dislocation showed similar reaching errors in the parasagittal $(0.14 \mathrm{~cm}$ more; $P=0.999)$ and oblique (0.36 cm less; $P=0.999)$ planes, but larger reaching errors (3.19 cm more, $P=0.437)$ in the frontal plane when compared with healthy controls. The main effect for target height was statistically significant $\left(F_{2,46}=11.01, P<0.001\right)$. Post hoc comparisons showed reaching accuracy to the bottom target was better than to the middle $(P<0.001)$ and top $(P=0.004)$ targets. The main effect for target plane was also statistically significant $\left(F_{2,46}=26.32, P<0.001\right)$. Post hoc comparisons showed reaching accuracy to the parasagittal plane was better than to the oblique $(P<0.001)$ and frontal $(P<0.001)$ planes. 


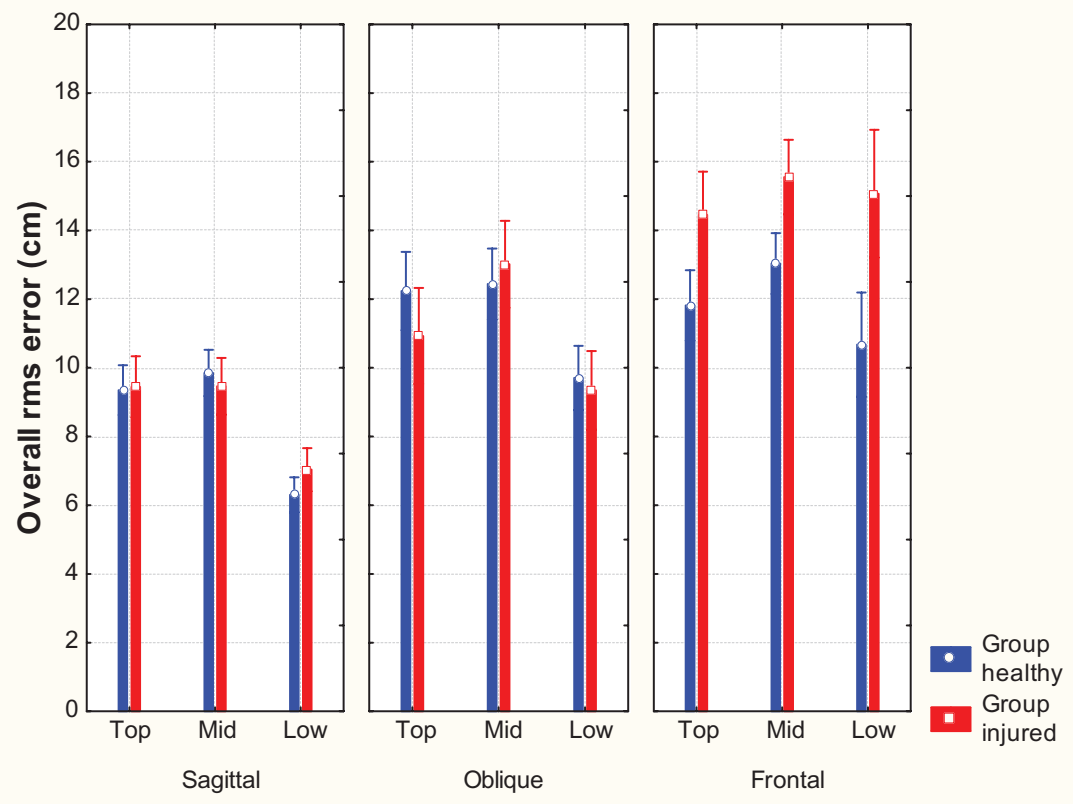

Figure 2 Mean root mean square errors of participants with healthy shoulders and participants with injured shoulders while reaching to targets at three different heights (top, mid, bottom) in three different planes (sagittal, oblique, frontal).

Note: Error bars denote one standard deviation.

Participants with a history of traumatic anterior shoulder dislocation showed significantly less external rotation when compared with participants with healthy shoulders during kinesthetically guided reaching $\left(F_{1,23}=7.54, P=0.011\right.$, Figure 3). The group $\times$ plane $\times$ height $\left(F_{4,92}=0.25, P=0.104\right)$, plane $\times$ height $\left(F_{4,92}=2.01, P=0.075\right)$, group $\times$ height $\left(F_{2,46}=0.82, P=0.383\right)$, and group $\times$ plane $\left(F_{2,46}=0.23\right.$, $P=0.722)$ interactions did not reach statistical significance.
The main effect for target height was statistically significant $\left(F_{2,46}=87.87, P<0.001\right)$. Post hoc comparisons showed that participants showed larger shoulder rotation angles when reaching to the high targets than to the middle $(P=0.001)$ and low targets $(P<0.001)$. The main effect for target plane was also statistically significant $\left(F_{2,46}=6.34, P=0.003\right)$. Post hoc comparisons showed participants had less shoulder rotation angle when reaching to targets in the parasagittal
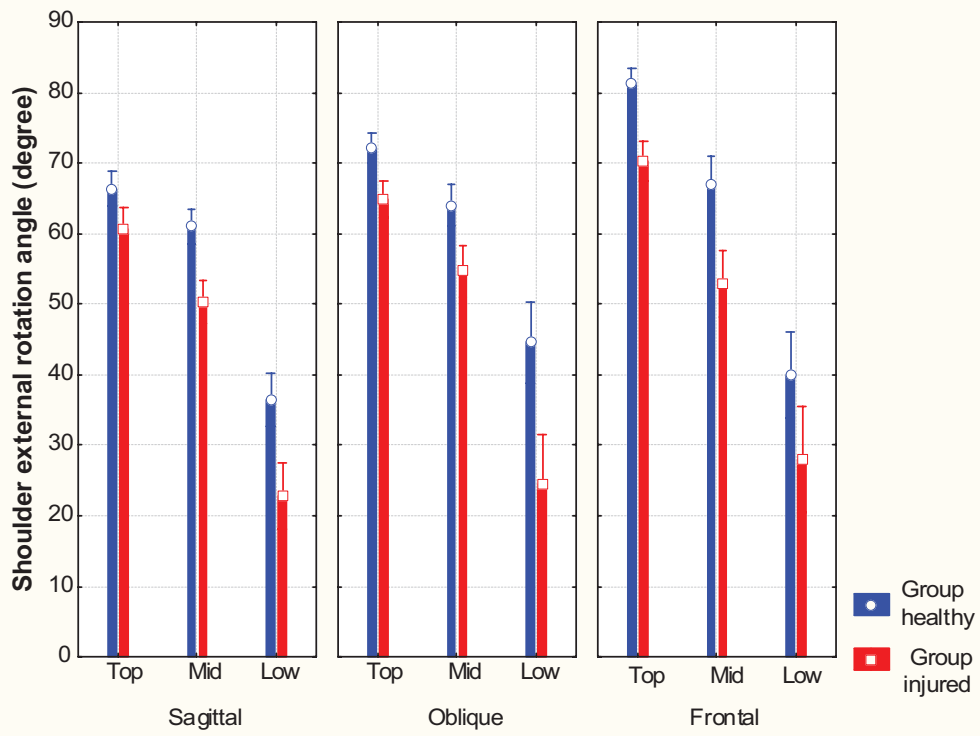

Figure 3 Mean shoulder external rotation angle of participants with healthy shoulders and participants with injured shoulders while reaching to targets at three different heights (top, mid, bottom) in three different planes (sagittal, oblique, frontal).

Note: Error bars denote one standard deviation. 
plane than to targets in the oblique $(P=0.047)$ and frontal planes $(P=0.001)$.

\section{Discussion}

\section{Reaching accuracy}

Our findings support the hypothesis that those with a history of traumatic anterior shoulder dislocation actively reach to visually specified and remember targets in space as accurately as those with healthy shoulders, with a group difference of only $1 \mathrm{~cm}$ in overall root mean square errors in this study. This investigation indicates that individuals with injured shoulders have sufficient kinesthetic information to point as accurately to visually specified targets in space as individuals with healthy shoulders. That is, an individual with an injured shoulder is able to transform visually specified target locations into an upper limb configuration that enables them to place the hand near the target location.

Further analysis showed that kinesthetically guided reaching accuracy depended strongly on the target location. Participants showed the smallest reaching errors in the sagittal plane and the largest errors in the frontal plane. Although the group differences did not reach significance in any of the three planes, participants with injured shoulders showed larger errors than controls in the frontal plane $(3.2 \mathrm{~cm}$ difference) than in the sagittal $(0.1 \mathrm{~cm}$ difference $)$ and oblique ( $0.4 \mathrm{~cm}$ difference) planes. Subconscious fear of shoulder redislocation while positioning the shoulder near end-range of external rotation with abduction (to targets in the frontal plane) may account for this observation. Moreover, both participants with injured and healthy shoulders showed the smallest errors while reaching to the low targets. Because the low targets were located much closer to the index finger at its resting position than the other two targets, participants were able to view both the target and the hand at the same time before initiating the movement, thereby enhancing the reaching accuracy.

\section{Shoulder rotation}

Participants with a history of traumatic anterior shoulder dislocation pointed to the remembered location by adopting a different reaching strategy with different upper limb configurations, presumably to protect the injured shoulder near vulnerable positions. In the present work, participants with injured shoulders averaged external rotation angles which were 12 degrees smaller than for participants with intact shoulders. To our surprise, smaller shoulder external rotations were consistent for all targets at different heights and in different planes, not only in vulnerable shoulder positions. The lack of shoulder external rotation was not due to physical restraints nor discomfort, because all participants achieved more than 90 degrees of shoulder external rotation and had normal shoulder external rotation muscle strength in the screening test. The results of this investigation suggest that individuals with injured shoulders adapted a different pointing strategy with less shoulder external rotation, perhaps to protect the injured shoulder from further injury.

\section{Limitations}

We only recruited subjects with a confirmed diagnosis of shoulder dislocation from a university medical center and excluded subjects with only partial dislocation or subluxation and weakness of the shoulder musculature. We tried to enhance the statistical power by matching the cohort control group (ie, gender, age, dominant/nondominant arm ratio, and fitness level). However, the small sample size in the current study could only detect a large effect size. Further investigation with a larger sample size would be beneficial.

For subjects with healthy shoulders, speed would not significantly impact three-dimensional reaching accuracy unless the speed is higher ${ }^{21,22}$ or lower $^{21}$ than normal. In the present study, the participants were asked to reach with natural/comfortable speed. Further research should consider standardizing the movement speed of participants. Considering that accurate control of hand location is crucial in many sports that require a fast movement velocity (eg, baseball, football), future studies that compare endpoint reaching accuracy and upper limb kinematics during upper extremity movement at high velocities would also be beneficial.

\section{Conclusion}

The purpose of this investigation was to determine whether individuals with a history of traumatic anterior shoulder dislocation show larger endpoint errors than individuals with healthy shoulders in a kinesthetically guided voluntary reaching task that resembled functional activities. The second aim of the study was to determine if participants in both groups use similar shoulder orientation at the endpoint of such reaches. Participants with injured shoulders can point to visually specified and remembered targets in space as accurately as those with healthy shoulders. In addition, participants with injured shoulders used a different reaching strategy (less external rotation) to point to the visually remembered targets compared with those with healthy shoulders, perhaps to protect their injured shoulders. Even without surgical repair, individuals with a history of traumatic anterior shoulder dislocation have sufficient kinesthetic information about 
their upper limb orientation to point accurately to visually specified targets in space. Therefore, shoulder instability did not cause significant functional impairment of the upper limb. Individuals with a history of anterior shoulder dislocation were able to engage appropriate motor control to modify their upper limb configurations (with less external rotation), presumably to protect their shoulders from recurrent injury.

\section{Disclosure}

The authors report no conflicts of interest in this work.

\section{References}

1. Brenneke SL, Reid J, Ching RP, Wheeler DL. Glenohumeral kinematics and capsulo-ligamentous strain resulting from laxity exams. Clin Biomech (Bristol, Avon). 2000;15:735-742.

2. Itoi $\mathrm{E}, \mathrm{Hsu} \mathrm{HC}, \mathrm{An} \mathrm{KN}$. Biomechanical investigation of the glenohumeral joint. J Shoulder Elbow Surg. 1996;5:407-424.

3. Turkel SJ, Panio MW, Marshall JL, Girgis FG. Stabilizing mechanisms preventing anterior dislocation of the glenohumeral joint. $J$ Bone Joint Surg Am. 1981;63:1208-1217.

4. von Eisenhart-Rothe RM, Jager A, Englmeier KH, Vogl TJ, Graichen H. Relevance of arm position and muscle activity on three-dimensional glenohumeral translation in patients with traumatic and atraumatic shoulder instability. Am J Sports Med. 2002;30:514-522.

5. Davies GJ, Dickoff-Hoffman S. Neuromuscular testing and rehabilitation of the shoulder complex. J Orthop Sports Phys Ther. 1993;18:449-458.

6. Lephart SM, Warner JJP, Borsa PA, Fu FH. Proprioception of the shoulder joint in healthy, unstable, and surgically repaired shoulders. J Shoulder Elbow Surg. 1994;3:371-380.

7. Myers JB, Lephart SM. The role of the sensorimotor system in the athletic shoulder. J Athl Train. 2000;35:351-363.

8. Nyland JA, Caborn DN, Johnson DL. The human glenohumeral joint. A proprioceptive and stability alliance. Knee Surg Sports Traumatol Arthrosc. 1998;6:50-61.

9. Smith RL, Brunolli J. Shoulder kinesthesia after anterior glenohumeral joint dislocation. Phys Ther. 1989;69:106-112.

10. Zuckerman JD, Gallagher MA, Cuomo F, Rokito A. The effect of instability and subsequent anterior shoulder repair on proprioceptive ability. J Shoulder Elbow Surg. 2003;12:105-109.

11. Rokito AS, Birdzell MG, Cuomo F, Di Paola MJ, Zuckerman JD. Recovery of shoulder strength and proprioception after open surgery for recurrent anterior instability: a comparison of two surgical techniques. J Shoulder Elbow Surg. 2010;19:564-569.

12. Hung YJ, Darling WG. Shoulder position sense during passive matching and active positioning tasks in individuals with anterior shoulder instability. Phys Ther. 2012;92:563-573.

13. Gandevia SC, McCloskey DI, Burke D. Kinaesthetic signals and muscle contraction. Trends Neurosci. 1992;15:62-65.

14. McCloskey DI. Kinesthetic sensibility. Physiol Rev. 1978;58:763-820.

15. Suprak DN, Osternig LR, van Donkelaar P, Karduna AR. Shoulder joint position sense improves with external load. J Mot Behav. 2007;39: $517-525$.

Orthopedic Research and Reviews

\section{Publish your work in this journal}

Orthopedic Research and Reviews is an international, peer-reviewed, open access journal focusing on the patho-physiology of the musculoskeletal system, trauma, surgery and other corrective interventions to restore mobility and function. Advances in new technologies, materials, techniques and pharmacological agents are particularly welcome. The journal welcomes
16. Brindle TJ, Uhl TL, Nitz AJ, Shapiro R. The influence of external loads on movement precision during active shoulder internal rotation movements as measured by 3 indices of accuracy. J Athl Train. 2006;41:60-66.

17. Gandevia SC, Smith JL, Crawford M, Proske U, Taylor JL. Motor commands contribute to human position sense. J Physiol. 2006;571:703-710.

18. Walsh LD, Hesse CW, Morgan DL, Proske U. Human forearm position sense after fatigue of elbow flexor muscles. J Physiol. 2004;558:705-715.

19. Erickson RI, Karduna AR. Three-dimensional repositioning tasks show differences in joint position sense between active and passive shoulder motion. J Orthop Res. 2012;30:787-792.

20. Fuentes CT, Bastian AJ. Where is your arm? Variations in proprioception across space and tasks. J Neurophysiol. 2010;103:164-171.

21. Messier J, Adamovich S, Berkinblit M, Tunik E, Poizner H. Influence of movement speed on accuracy and coordination of reaching movements to memorized targets in three-dimensional space in a deafferented subject. Exp Brain Res. 2003;150:399-416.

22. Adamovich S, Berkinblit M, Smetanin B, Fookson O, Poizner H. Influence of movement speed on accuracy of pointing to memorized targets in 3D space. Neurosci Lett. 1994;172:171-174.

23. Adamovich SV, Berkinblit MB, Fookson O, Poizner H. Pointing in $3 \mathrm{D}$ space to remembered targets. I. Kinesthetic versus visual target presentation. J Neurophysiol. 1998;79:2833-2846.

24. Soechting JF, Flanders M. Sensorimotor representations for pointing to targets in three-dimensional space. $J$ Neurophysiol. 1989;62:582-594.

25. Darling WG, Miller GF. Transformations between visual and kinesthetic coordinate systems in reaches to remembered object locations and orientations. Exp Brain Res. 1993;93:534-547.

26. Darling WG, Bartelt R, Pizzimenti MA, Rizzo M. Spatial perception errors do not predict pointing errors by individuals with brain lesions. J Clin Exp Neuropsychol. 2008;30:102-119.

27. Lo IK, Nonweiler B, Woolfrey M, Litchfield R, Kirkley A. An evaluation of the apprehension, relocation, and surprise tests for anterior shoulder instability. Am J Sports Med. 2004;32:301-307.

28. Bjorklund M, Crenshaw AG, Djupsjobacka M, Johansson H. Position sense acuity is diminished following repetitive low-intensity work to fatigue in a simulated occupational setting. Eur J Appl Physiol. 2000;81:361-367.

29. Skinner HB, Barrack RL, Cook SD. Age-related decline in proprioception. Clin Orthop Relat Res. 1984:208-211.

30. Kaplan FS, Nixon JE, Reitz M, Rindfleish L, Tucker J. Age-related changes in proprioception and sensation of joint position. Acta Orthop Scand. 1985;56:72-74.

31. Milne AD, Chess DG, Johnson JA, King GJ. Accuracy of an electromagnetic tracking device: a study of the optimal range and metal interference. J Biomech. 1996;29:791-793.

32. Meskers CG, Fraterman H, van der Helm FC, Vermeulen HM, Rozing PM. Calibration of the "Flock of Birds" electromagnetic tracking device and its application in shoulder motion studies. J Biomech. 1999;32:629-633.

33. Meskers CG, Vermeulen HM, de Groot JH, van Der Helm FC, Rozing PM. 3D shoulder position measurements using a six-degreeof-freedom electromagnetic tracking device. Clin Biomech (Bristol, Avon). 1998;13:280-292.

\section{Dovepress}

original research, clinical studies, reviews \& evaluations, expert opinion and commentary, case reports and extended reports. The manuscript management system is completely online and includes a very quick and fair peer-review system, which is all easy to use. Visit http://www.dovepress. com/testimonials.php to read real quotes from published authors. 